\title{
The fecal microbiota composition of boar Duroc, Yorkshire, Landrace and Hampshire pigs
}

\author{
Yingping Xiao ${ }^{1,2, a}$, Kaifeng Li $^{1,2,3, a}$, Yun Xiang ${ }^{4}$, Weidong Zhou ${ }^{5}$, Guohong Gui ${ }^{1,2}$, and Hua Yang ${ }^{1,2, *}$
}

* Corresponding Author: Hua Yang Tel: +86-0571-8640-4005, Fax: +86-0571-8640-4005, E-mail: yanghua806@hotmail.com

${ }^{1}$ Institute of Quality and Standard for Agro-products, Zhejiang Academy of Agricultural Sciences, Hangzhou 310021, China

2 State Key Laboratory Breeding Base for Zhejiang Sustainable Pest and Disease Control, Zhejiang Academy of Agricultural Sciences, Hangzhou 310021, China

${ }^{3}$ College of Animal Sciences, Zhejiang University, Hangzhou 310058, China

${ }^{4}$ Animal Husbandry and Veterinary Institute, Jinhua Academy of Agricultural Sciences, Jinhua 321017, China

${ }^{5}$ Animal Husbandry and Veterinary Institute, Zhejiang Academy of Agricultural Sciences, Hangzhou 310021, China

a These authors contributed equally to this work. Submitted Sept 30, 2016; Revised Dec 16, 2016; Accepted Feb 16, 2017
Objective: To investigate the effect of host genetics on gut microbial diversity, we performed a structural survey of the fecal microbiota of four purebred boar pig lines: Duroc, Landrace, Hampshire, and Yorkshire.

Methods: The V3-V4 regions of the 16S rRNA genes were amplified and sequenced.

Results: A total of 783 operational taxonomic units were shared by all breeds, whereas others were breed-specific. Firmicutes and Bacteroidetes dominated the majority of the fecal microbiota; Clostridia, Bacilli, and Bacteroidia were the major classes. Nine predominant genera were observed in all breeds and eight of them can produce short-chain fatty acids. Some bacteria can secrete cellulase to aid fiber digestion by the host. Butyric, isobutyric, valeric, and isovaleric acid levels were highest in Landrace pigs, whereas acetic and propionic acid were highest in the Hampshire breed. Heatmap was used to revealed breed-specific bacteria. Principal coordinate analysis of fecal bacteria revealed that the Landrace and Yorkshire breeds had high similarity and were clearly separated from the Duroc and Hampshire breeds.

Conclusion: Overall, this study is the first time to compare the fecal microbiomes of four breeds of boar pig by high-throughput sequencing and to use Spearman's rank correlation to analyze competition and cooperation among the core bacteria.

Keywords: Boar; Breed; Fecal Microbiota; High-throughput Sequencing; Short-chain Fatty Acids

\section{INTRODUCTION}

The pig gastrointestinal tract harbors thousands of species of bacteria, whose composition and relative proportions vary depending on the animal breed, animal age, and nutritional and environmental factors [1]. Gut bacteria influence nutrient absorption and the health of the host [2,3], such as vitamin synthesis and short-chain fatty acid (SCFA) production [4]. In particular, genetic background is strongly associated with the host's gut microbial taxa and characteristics $[2,4]$.

Hampshire, Landrace, Duroc, and Yorkshire are the most frequently used pigs in commercial production and have favorable growth performance. These breeds had various origins. Duroc pigs are red, muscular pigs that originated in America. Yorkshire, also known as Large White, is a breed originating in Yorkshire. The Hampshire pig has a black body with a white band covering the front legs and originated from America. The Landrace breed was developed in Denmark by crossing the native pig with the Large White and has white skin. Landrace has bigger size and faster growing rate but low ability of resistance to disease [5]. Overall, male pigs utilize feed more efficiently and have profitable production characteristics, but few studies have compared the fecal bacteria compositions of these four boar breeds [6]. In this study, the four purebred boar pig lines were housed in a controlled environment and fed the same diet. Due to their distinct genetic backgrounds, the four breeds of pigs exhibit differences in morphology, reproduction and digestive capacity. Therefore, these breeds of pigs may exhibit breed-specific microbial diversity [2,7]. The 
Illumina Sequencing platform yields longer deoxyribonucleic acid (DNA) reads at lower cost compared to other platforms and provides more abundant information on bacterial communities [3]. However, given the diverse relationships between boar pig properties and gut microbiome function, further study is needed to reveal the contribution of gut microbiome and its metabolic products to an animal's economic characteristics.

To assess the influence of pig breed on shaping the gut microbiota and to determine whether these breeds share core bacteria or have breed-specific bacteria, we chose twenty-three boar pigs (Duroc $=6$, Hampshire $=4$, Landrace $=7$, Yorkshire $=6$ ) representing four breeds and diverse genetic backgrounds. With the help of sequence statistics, we identified ten predominant genera that co-occurred in all pig breeds and several breed-specific bacteria that may be potentially functional microorganism to pig breeds.

\section{MATERIALS AND METHODS}

\section{Sample collection and DNA extraction}

Fresh fecal samples were collected from four purebred boar pigs approximately 300 days of age. Pigs were raised by a method approved by the Animal Care and Use Committee of ZAAS (Approval number: ZAAS-2015-008). The four breeds were raised in under standard American facility farming (Tonglu, Zhejiang, China), and all pigs received the same primary diet based on corn and soybean without antibiotics. All pigs were grown in the same hogpen. The temperature and humidity of the house were controlled at comfortable levels (temperature was $16^{\circ} \mathrm{C}$ to $21^{\circ} \mathrm{C}$ and humidity was $60 \%$ to $80 \%$ ). All samples were collected on the same day in the morning before feeding and frozen in liquid nitrogen before immediate transport to the laboratory. Total genomic DNA was extracted from the samples using the QIAamp DNA Stool Mini Kit (QIAGEN, CA, Hamburg, Germany) according to the manufacturer's instructions [2]. The DNA concentration and purity were monitored on $1 \%$ agarose gels. The quantity of DNA was measured using a NanoDrop 1000 spectrophotometer, and the DNA concentration was diluted to $1 \mathrm{ng} / \mu \mathrm{L}$ using sterile water.

\section{Polymerase chain reactions amplicon production and high-throughput sequencing}

The V3-V4 distinct regions of $16 \mathrm{~S}$ rRNA (ribosome ribonucleic acid) genes were amplified using specific primers $515^{\prime} \mathrm{F}$ (GTGBC AGCMGCCGCGGTAA) and 805R (GGACTACHVGGGTWT CTAAT) with barcodes [8]. The polymerase chain reactions (PCRs) were carried out in triplicate in a total volume of $25 \mu \mathrm{L}$ containing $5 \mu \mathrm{M}$ of each primer, $10 \mathrm{ng}$ of DNA template, $4 \mu \mathrm{L}$ $1 \times$ FastPfu buffer, $2.5 \mathrm{mM}$ dNTPs, and $0.4 \mu \mathrm{L}$ of FastPfu polymerase (TransGen Biotech, Beijing, China). The following PCR cycles were used: initial denaturation at $94^{\circ} \mathrm{C}$ for $5 \mathrm{~min}, 30$ cycles at $94^{\circ} \mathrm{C}$ for $50 \mathrm{~s}, 55^{\circ} \mathrm{C}$ for $30 \mathrm{~s}$, and $72^{\circ} \mathrm{C}$ for $50 \mathrm{~s}$, and a final extension at $72^{\circ} \mathrm{C}$ for $6 \mathrm{~min}$. Then the mixture of PCR products was purified using the AxyPrep DNA Gel Extraction Kit (Axygen, Union City, CA, USA). Amplicons produced from different samples were sent to a commercial company (Majorbio, Shanghai, China) for sequencing on an Illumina HiSeq 2500 platform.

\section{Analysis of short-chain fatty acids and $\mathrm{pH}$}

The four breeds pigs' fecal samples was mixed ( $1 \mathrm{~g}$ dry matter) with ten times Deionized Water. Then the mixture were centrifuged $(10,000 \mathrm{rpm}$ for $10 \mathrm{~min})$ and $1,000 \mu \mathrm{L}$ of the supernatant was added with $200 \mu \mathrm{L}$ of crotonic acid (internal standard). Sample was injected on the gas chromatography-column and the content of SCFAs were analyzed using the method described by Yang et al [2].

\section{Data analysis}

First, the paired-end reads were assigned to samples based on their unique barcodes and truncated by removing the barcode and primer sequences. Paired-end reads were merged using FLASH (V1.2.7). The spliced sequences were called raw tags. Quality filtering of the raw tags was performed under specific filtering conditions to obtain high-quality clean tags according to the Qiime (V1.7.0) quality controlled process. The tags were compared with the reference database using the UCHIME algorithm to detect chimera sequences, and then the chimera sequences were removed to finally obtain the Effective Tags. Community analysis was performed using the Uparse software (v7.0.1001). Richness and diversity indices were produced using Mothur, and sets of sequences with $\geq 97 \%$ identity were defined as operational taxonomic units (OTUs). The representative sequences were distributed into phyla and genera according to the SILVA bacteria database (SILVA version 108, Silva, Bremen, Germany) following the Bayesian approach and using a cutoff of $97 \%$. Subsequent analyses of alpha diversity and beta diversity were all performed basing on this output normalized data. Clusters were generated using a dissimilarity cutoff of 3\% for calculating the Shannon, Chao 1 richness and abundance-based coverage estimators (ACE) using the Mothur package [9].

\section{RESULTS}

\section{Bacterial diversity and composition of the four boar pig breeds}

In this study, high-throughput sequencing technology was adopted, and a total of 194,813 valid sequence reads were generated from Duroc $\left(47,796^{\mathrm{b}}\right)$, Hampshire $\left(47,535^{\mathrm{b}}\right)$, Landrace $\left(50,842^{\mathrm{a}}\right)$ and Yorkshire $\left(48,640^{\mathrm{b}}\right)$. OTUs with a cut-off of $97 \%$ identity were then identified in Duroc $\left(994^{\mathrm{ab}}\right)$, Hampshire $\left(930^{\mathrm{b}}\right)$, Landrace $\left(1,046^{\mathrm{a}}\right)$, and Yorkshire $\left(1,028^{\mathrm{a}}\right)$ (Table 1$)$. The Shannon diversity index, ACE and Chaol were used to compare the bacterial diversities among the four boar pig breeds. Duroc $\left(6.29^{\mathrm{a}}\right)$ exhibited the highest Shannon diversity index and had a more diverse bacterial community compared to Hampshire $\left(6.20^{\mathrm{a}}\right)$, Landrace $\left(6.08^{\mathrm{ab}}\right)$, 
Table 1. Diversity indices and summary of the 165 rRNA gene pyrosequencing data"

\begin{tabular}{|c|c|c|c|c|}
\hline Measurement & Duroc $(n=6)$ & Hampshire $(n=4)$ & Landrace $(n=6)$ & Yorkshire $(n=7)$ \\
\hline Valid reads & $47,796^{b}$ & $47,535^{b}$ & $50,842^{\mathrm{a}}$ & $48,640^{b}$ \\
\hline OTUs & $994^{\mathrm{ab}}$ & $930^{b}$ & $1,046^{\mathrm{a}}$ & $1,028^{\mathrm{a}}$ \\
\hline Shannon diversity index & $6.29^{\mathrm{a}}$ & $6.20^{\mathrm{a}}$ & $6.08^{\mathrm{ab}}$ & $5.85^{b}$ \\
\hline Chao 1 estimator of species richness & $772^{\mathrm{a}}$ & $733^{b}$ & $763^{\mathrm{a}}$ & $744^{b}$ \\
\hline ACE estimator of species richness & $773^{\mathrm{a}}$ & $742^{b}$ & $774^{\mathrm{a}}$ & $754^{b}$ \\
\hline
\end{tabular}

OTUs, operational taxonomic units; ACE, abundance-based coverage estimator.

1) Calculations were performed based on the OTU definition at $>97 \%$ sequence identity. The numbers in the table are the average value for each breed.

${ }^{a b}$ Means in the same row with different superscript letters differ significantly $(p<0.05)$.

and Yorkshire $\left(5.85^{\mathrm{b}}\right)$. In addition, Hampshire contained a larger number of less-abundant bacteria than Landrace based on its greater Shannon index but lower ACE richness. The values of the Shannon and ACE indexes suggest that most of the fecal bacteria were captured (Table 1).

At the phylum level, Firmicutes and Bacteroidetes dominated the majority of the fecal microbiota regardless of breed. Other phyla were Proteobacteria, Spirochetes, Euryarchaeota, Actinobacteria, Fibrobacteres, Cyanobacteria, Verrucomicrobia, and Tenericutes. However, the bacterial community compositions of the breeds differed. For example, the proportion of the phylum Fimicutes was greater in the feces of Duroc (81.7\%), Landrace (83.2\%), and Yorkshire (87.0\%) than in Hampshire (74.2\%) (p $=0.064,0.03,0.003$, respectively). By contrast, the proportion of the phylum Bacteroidetes was higher in the feces of Hampshire (22.2\%) than in Duroc (14.8\%), Landrace (12.5\%), and Yorkshire (9.13\%) $(\mathrm{p}=0.065,0.019,0.002$, respectively) (Figure 1).

At the class level, Clostridia, Bacilli, and Bacteroidia dominated the majority of the fecal microbiota regardless of breed. Other abundant classes were Gammaproteobacteria, Spirochaetes, Methanobacteria, Erysipelotrichi, Coriobacteriia, Fibrobacteria, and Betaproteobacteria. However, differentially abundant bacterial communities were observed among the four breeds. For example, the proportion of Clostridia was much lower in the feces of Hampshire (52.3\%) than in Duroc (59.9\%), Landrace (66.1\%), and Yorkshire $(70.6 \%)(\mathrm{p}=0.021,0.012,0.001$, respectively). The

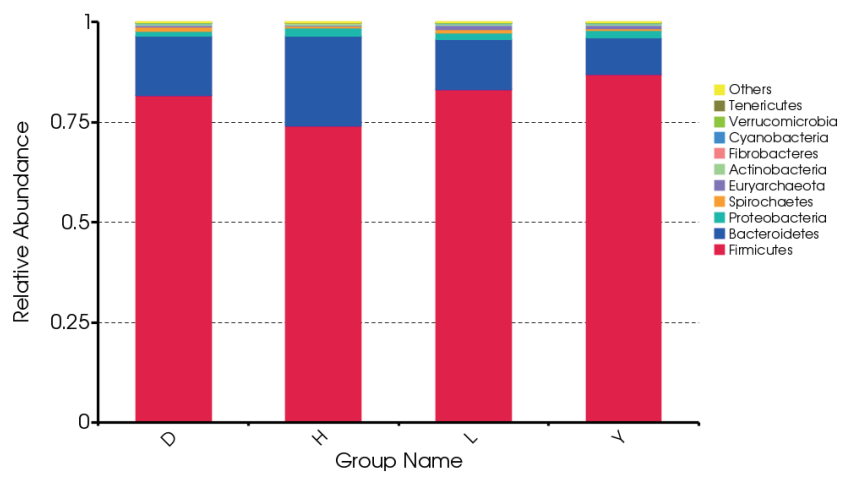

Figure 1. Distribution of bacterial phyla and their abundance among the fecal microbiota of the four boar pig breeds. D, Duroc; H, Hampshire; L, Landrace; Y, Yorkshire. proportion of Bacteroidia was greater in the feces of Hampshire (22.2\%) than in Duroc (14.8\%), Landrace (12.5\%), and Yorkshire (9.13\%) $(\mathrm{p}=0.065,0.019,0.002$, respectively) (Supplementary Figure S1).

At the genus level, we calculated the most abundant nine genera in the fecal microbiota of all breeds of pigs as core bacteria that accounted for $44.82 \%$ of the total sequences (Figure 2; Supplementary Figure S2). Most of the core genera were Firmicutes, except Prevotella and Bacteroides, which belong to Bacteroidetes. Similar to a previous report, Prevotella and Streptococcus were the most abundant genera [10]. Streptococcus (14.6\%) and Prevotella (14.3\%) were more abundant in the fecal microbiota of Hampshire pigs than the other breeds $(\mathrm{p}<0.05)$. A previous study showed that Prevotella decreased from $30 \%$ of all bacteria to $4.0 \%$ as pigs aged; these bacteria degrade mucin and xylan, which might be helpful in feed digestion $[11,12]$. The abundance of the genus Lactobacillus (11.1\%) was markedly greater in Duroc pigs than in the other three breeds $(\mathrm{p}<0.05)$, in contrast to a previous study that observed that the abundance of Lactobacillus was highest in Landrace pigs [7]. The abundance of the Clostridium genus differed among the four breeds and was highest in Yorkshire (14.2\%) and lowest in Hampshire $(6.67 \%, \mathrm{p}<0.05)$. The Turicibacter genus was more abundant in both Landrace (3.81\%) and Yorkshire (3.87\%, $\mathrm{p}<0.05)$ than the other two breeds. The Oscillospira genus was more abundant in Landrace (3.07\%) but less abundant in Hampshire $(2.01 \%, \mathrm{p}<0.05)$. The Coprococcus genus was more abundant

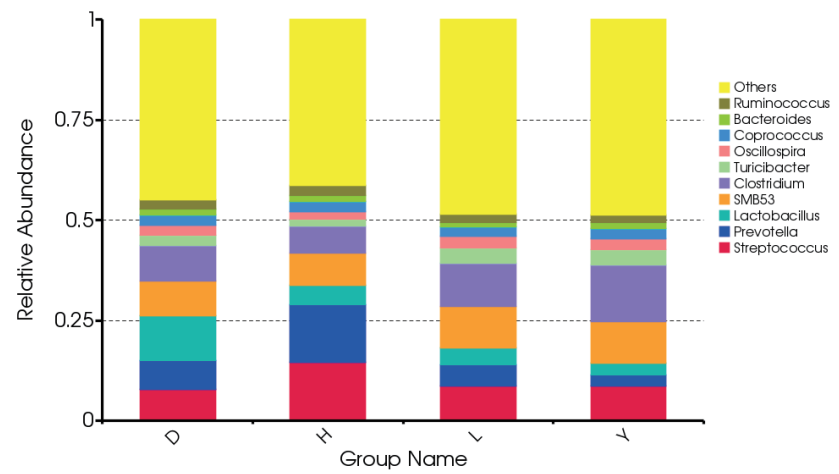

Figure 2. Distribution of bacterial genera and their abundance among the fecal microbiota of the four boar pig breeds. D, Duroc; H, Hampshire; L, Landrace; Y, Yorkshire. 
Streptococcus Prevotella Lactobacillus Clostridium Turicibacter Oscillospira Coprococcus Bacteroides Ruminococcus

\begin{tabular}{lrrrrrrrrr}
\hline Streptococcus & 1 & 0.218 & 0.28 & -0.365 & -0.499 & -0.346 & 0.007 & 0.243 & -0.067 \\
\hline Prevotella & 0.218 & 1 & 0.458 & -0.723 & -0.705 & -0.421 & 0.442 & 0.445 & 0.287 \\
\hline Lactobacillus & 0.28 & 0.458 & 1 & -0.6 & -0.688 & -0.275 & 0.261 & 0.312 & 0.038 \\
\hline Clostridiun & -0.365 & -0.723 & -0.6 & 1 & 0.679 & 0.398 & -0.216 & -0.597 & -0.073 \\
\hline Turicibacter & -0.499 & -0.705 & -0.688 & 0.679 & 1 & 0.372 & -0.428 & -0.594 & -0.156 \\
\hline Oscillospira & -0.346 & -0.421 & -0.275 & 0.398 & 0.372 & 1 & -0.202 & -0.222 & 0.137 \\
\hline Coprococcus & 0.007 & 0.442 & 0.261 & -0.216 & -0.428 & -0.202 & 1 & 0.448 & 0.27 \\
\hline Bacteroides & 0.243 & 0.445 & 0.312 & -0.597 & -0.594 & -0.222 & 0.448 & 1 & -0.035 \\
Ruminococcus & -0.067 & 0.287 & 0.038 & -0.073 & -0.156 & 0.137 & 0.27 & -0.035 & 1
\end{tabular}

Figure 3. Co-occurrence patterns among the nine core bacteria across the 23 samples as determined by Spearman's rank correlation analysis. The correlation coefficient ( $r$ ) indicates the extent to which the two genera co-occur. The different colors represent the range of $(r)$ from -1 to +1 . Orange indicates a perfect positive correlation, whereas green indicates a perfect negative correlation.

in Yorkshire (2.57\%) but less abundant in Landrace $(2.21 \%, \mathrm{p}<$ 0.05). Bacteroides was less abundant in Landrace whereas Ruminococcus was more abundant in Hampshire, but these differences were not significant.

Spearman's rank correlation test was used to analyze the cooccurrence patterns among the nine core bacteria (Figure 3). Some bacteria were positively correlated with each other. For example, Prevotella and Lactobacillus were significantly correlated with each other $($ rho $=0.458)$ and with other bacteria such as Coprococcus $(\mathrm{rho}=0.442,0.261)$, Bacteroides $(\mathrm{rho}=0.445,0.312)$ and Streptococcus (rho $=0.218,0.28$ ). Clostridium was positively correlated with Turicibacter $(\mathrm{rho}=0.679)$ but negatively correlated with the other six bacteria. In contrast to previous findings for human gut bacteria [13], co-occurrence between Prevotella and Ruminococcus was observed in the four pig breeds (rho $=0.287$ ), and we observed co-exclusion of Prevotella and Clostridium (rho $=-0.723)$, Turicibacter $($ rho $=-0.705)$ and Oscillospira $(r h o=$ -0.421). As shown in Figure 5, Streptococcus, Prevotella and Lactobacillus co-occurred and cooperated to suppress three other cooperative genera: Clostridium, Turicibacter, and Oscillospira. These results may reflect multiple mechanisms such as secretory products and competition for nutrients [14]. The contribution of these co-occurring genera and co-excluding genera as revealed in this study may have some fundamentally important function and must be researched deeply.

\section{SCFAs, $\mathrm{pH}$, and cellulose activity of the fecal microbiota} of the four pig breeds

We also measured the SCFAs, $\mathrm{pH}$, and cellulose activity of the fecal microbiota of the four breeds of pig (Figures 4, 5). The bu-

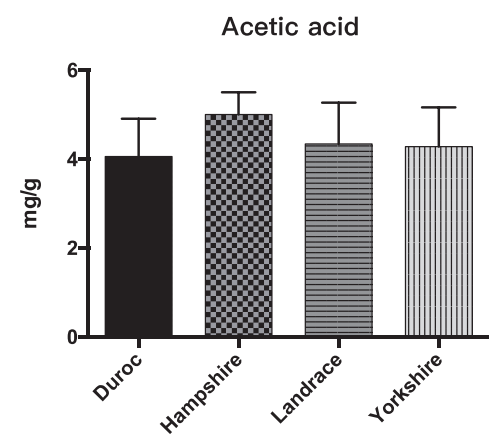

Isobutyric acid

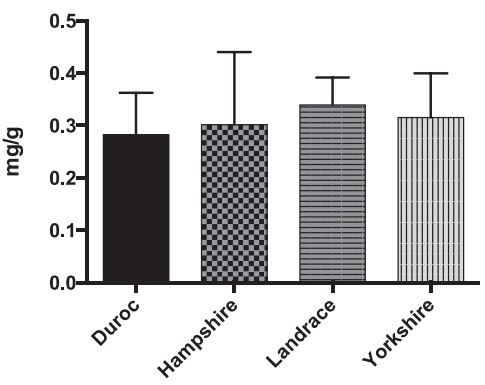

Propionic acid

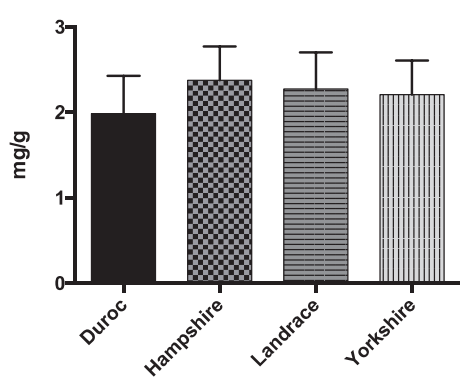

Valeric acid

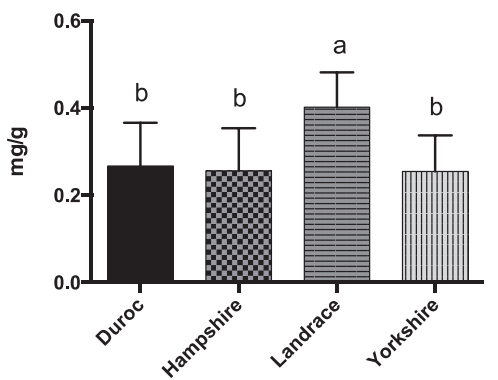

Butyric acid

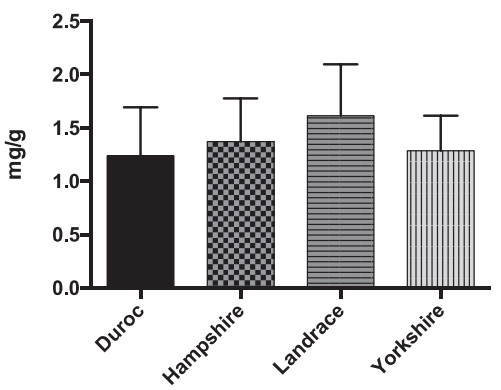

Isovaleric acid

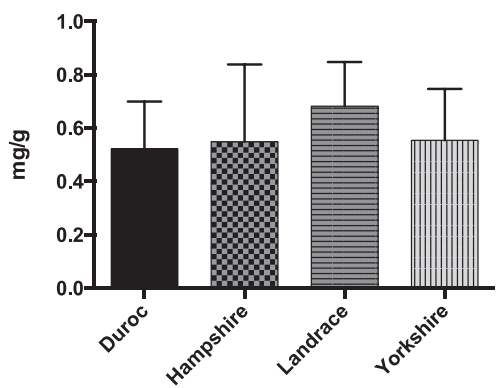

Figure 4. Distribution of short-chain fatty acids (SCFAs) of the four boar pig breeds. The error bars represent standard deviations. 
$\mathrm{pH}$

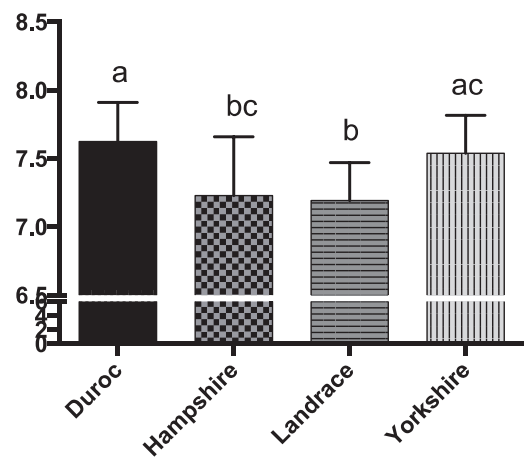

Cellulase $\mathrm{U} / \mathrm{g}$

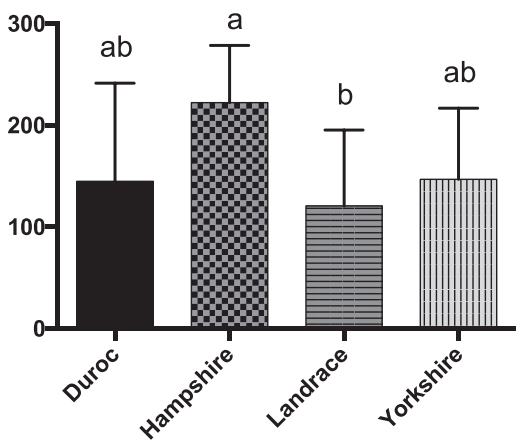

Figure 5. Distribution of pH and cellulase activity of the four boar pig breeds. The error bars represent standard deviations.

tyric, isobutyric, valeric, and isovaleric acid levels were higher in Landrace pigs than in the other three breeds, whereas acetic and propionic acid were highest in the Hampshire breed. These differences were associated with $\mathrm{pH}$ : $\mathrm{pH}$ was significantly lower for Hampshire and Landrace than for the other two breeds. Most mammals have a limited ability to produce cellulase and thus cannot digest fibers by themselves. Some gut bacteria of pigs, such as Clostridium, Bacillus, Ruminococcus, and Bacteroides, produce cellulases to help their host digest diet fibers.

\section{Different abundant bacteria and principal coordinate analysis of the four pig breeds}

The abundance distribution of the dominant 35 genera among the four breeds was displayed in a species abundance heatmap

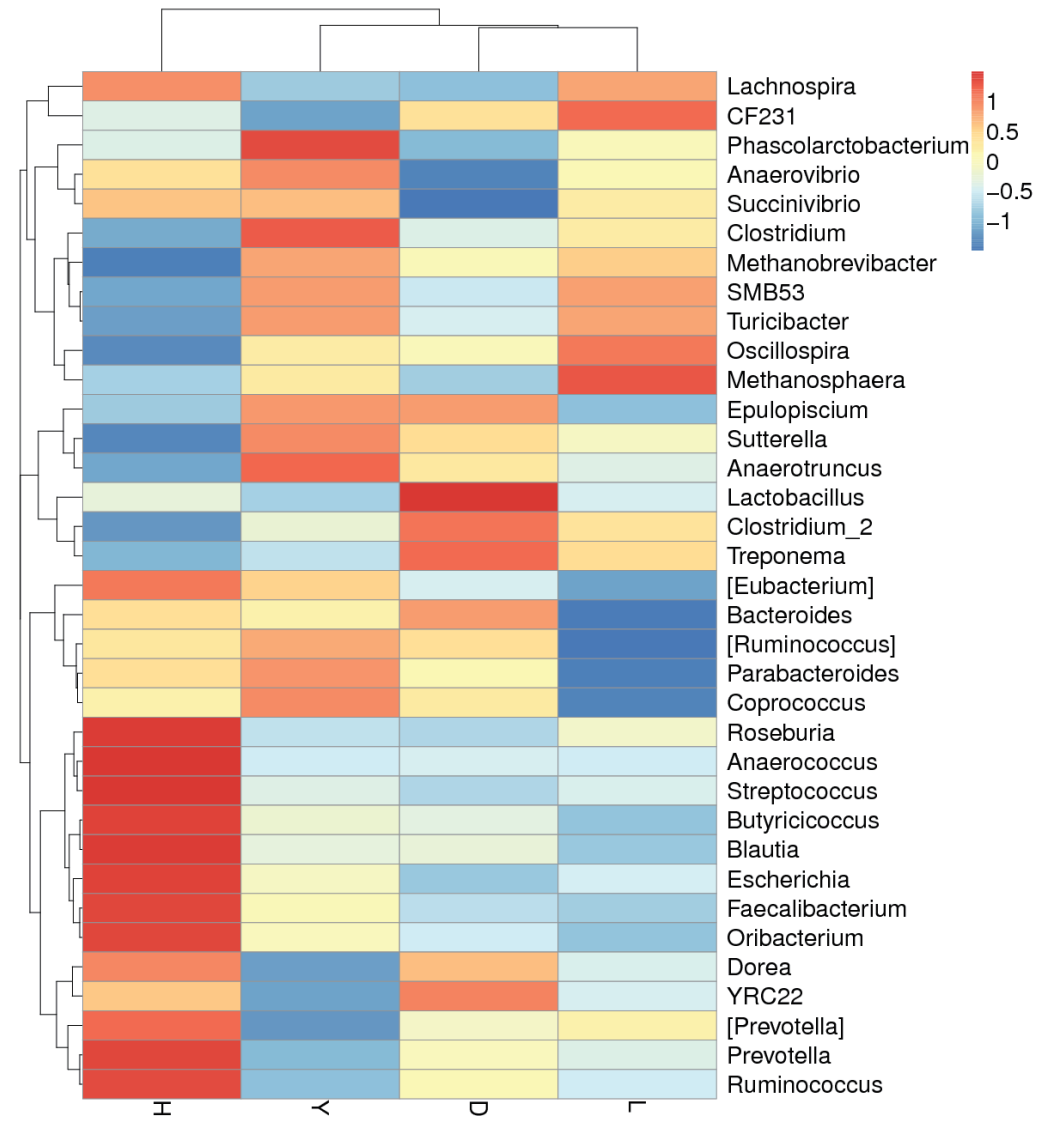

Figure 6. Species abundance heatmap showing normalized values of 35 differentially abundant genera of four boar pig breeds. Breed name is plotted on the X-axis, and the Y-axis represents the genus. The different colors represent the distance between the raw score and the mean population of the standard deviation. Red indicates that the $z$-value of a specific genus is larger than the mean, whereas blue indicates the opposite. D, Duroc; H, Hampshire; L, Landrace; Y, Yorkshire. 
(Figure 6). The information for the clustering results of the samples and taxa revealed whether the samples with similar processing were clustered or not and the similarity and difference between the samples. Although these 35 genera were common to the fecal microbiota of all of the pig breeds, the heatmap revealed several genera that exhibited variations between breeds. For example, the genera Streptococcus, Prevotella, Ruminococcus, Roseburia, Anaerococcus, Butyricicoccus, Blautia, Escherichia, Faecalibacterium, and Oribacterium were more abundant in Hampshire pig fecal microbiota. The genera Phascolarctobacterium and Clostridium were more abundant in Yorkshire. The genera Lactobacillus and Treponema were more abundant in Duroc, whereas Methanosphaera was more abundant in Landrace. Thus, the variations among the breeds were specific and functionally relevant.

Principal coordinate analysis (PCoA) is an ordination technique that is similar to PCA. PCoA identifies the main elements and structure from reduced multi-dimensional data series of eigenvalues and eigenvectors. The advantage of PCoA over PCA is that each ecological distance can be investigated. PCA identifies the main coordinates based on the similarity coefficient matrix of all samples, whereas PCoA is based on a distance matrix. Weighted UniFrac and Unweighted UniFrac are calculated to assist the PCoA analysis. Clustered samples represent high species composition similarity compared to separated samples. The fecal bacterial communities clustered according to different breeds (Figure 7). The microbiomes of the Landrace and Yorkshire breeds had high similarity but were clearly separated from those of the Duroc and Hampshire breeds, similar to a previous result [7].
According to the analysis results for OTU clustering and the research requirements, we normalized the OTU table and analyzed both the shared and unique information for different samples (groups). The Venn diagram (Supplementary Figure S4) revealed that the four breeds shared most OTUs (783), but some breedspecific OTUs were observed. For example, Duroc had 16 specific OTUs, Landrace had 41, Yorkshire had 27, and Hampshire had 11. The associations of these specific OTUs with pig genetics and gut ecology warrant further research.

\section{DISCUSSION}

Previous studies have suggested that pig breed affects the composition of Firmicutes, Bacteroidetes, and sulfate-reducing bacteria, which are higher in Chinese native pig breeds than in foreign breeds [2]. Both genetic and cage effects are significantly associated with microbiota composition [15]. Firmicutes and Bacteroidetes were abundant phyla in the fecal bacterial community at preweaning [16], post-weaning and four years of age [12] but changed as pigs aged. Firmicutes and Actinobacteria are more abundant in lean Gottingen minipigs than in lean Ossabaw minipigs [17]. Firmicutes and Bacteroidetes are also the two most abundant phyla in the healthy human gut microbiota, but the ratio of these two phyla varies among individuals $[4,18]$. A previous study reported similar results for the gut tract of other breeds of pig [12].

At class level, the result differs from the results of previous studies in which the dominant class was Bacteroidia followed by Clostridia for all breeds $[1,7]$. In addition, the proportion of Bacilli

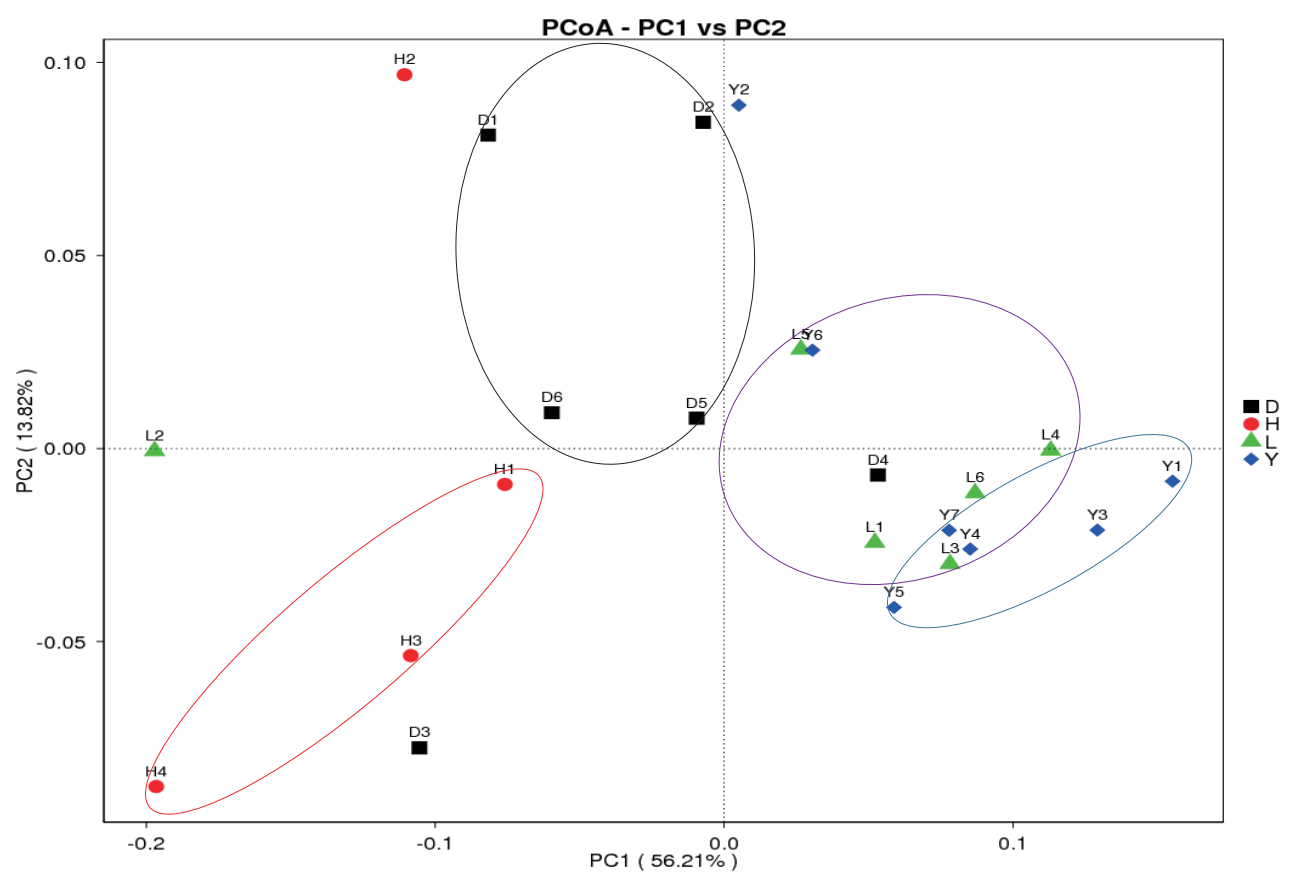

Figure 7. PCoA analysis based on Weighted UniFrac distance. Each point represents a sample. The first principal component is plotted on the X-axis, and the second principal component is plotted on the $\mathrm{Y}$-axis. The colors indicate different breeds. The percentage on each axis indicates the contribution to the discrepancy among samples. PCoA, principal coordinate analysis; D, Duroc; H, Hampshire; L, Landrace; Y, Yorkshire. 
was greater in the feces of Duroc (21.3\%) and Hampshire (21.2\%) than in Landrace (16.7\%) and Yorkshire (15.8\%). A previous study had showed that Bacilli and Clostridia were more abundant in obese Ossabaw minipigs, whereas Bacteroidia was more abundant in lean Ossabaw minipigs [17].

The differences in the abundance of genera among various breeds may be influenced by differences in gut function among specific breeds [19]. Functional analysis of Yorkshire pig fecal DNA extracts revealed that carbohydrate metabolism-associated bacteria reach $13 \%$ of the swine metagenome [11]. A previous study found that the genera Prevotella and Bacteroides were more abundant in obese Gottingen minipigs, whereas Clostridium was higher in lean Gottingen minipigs. However, another study reported that Prevotella had a greater abundance in lean Ossabaw minipigs, whereas Clostridium was more abundant in obese Ossabaw minipigs [17]. Similar clustering of bacteria in pigs of the same age was observed in different breeds of pigs [12]. Oscillospira, an enigmatic bacterial genus that has never been cultured and is positively correlated with leanness and health, has been detected frequently in the human gut [20]. From the Spearman's rank correlation, a perfect positive correlation indicates that these bacteria cooperated with each other, whereas perfect negative correlation represents suppression. Spearman's rank correlation provides valuable and complementary information.

Host genetics have an important influence on microbial diversity [19] and breed-specific bacteria [7]. Bacillus subtilis BY-2, which produces cellulose, has been isolated from the intestine of Tibetan pigs whose diet consists of forage grass [21], suggesting that the function of gut bacteria depends on the genome of the host breed. However, a deeper level of sequencing data is required to determine the specific metabolic functions that are conserved.

Previous studies have demonstrated that Landrace and Yorkshire are more genetically similar, with closer fecal microbiomes, whereas Duroc and Hampshire are genetically distant $[7,22]$. The microbial compositions of the Duroc, Landrace, and Yorkshire breeds were clustered in one group and differed from those of Chinese breeds such as Bama and Meishan sows [2]. The microbial communities varied between Gottingen and Ossabaw minipigs, which are models of obesity [17]. Research on mice has demonstrated that genetic distance correlates positively with microbiota distance; genetically similar mice have more similar bacterial compositions than do genetically distant mice [15]. A study of different Chinese ethnic groups indicated that Mongol, Tibetan, Zhuang, Han, Bai, Kazakh, and Uyghur ethnic groups were separated from each other significantly $(p=0.0001)$ [4].

SCFAs, including acetate, propionate, butyrate and pentanoic acid, are major anions in the gut and are absorbed rapidly by colonic epithelial cells. Acetate can enter systemic circulation and be used for lipogenesis; butyrate is the major energy source for colonocytes [23]. These small anions can improve gut barrier function and are associated with many metabolic activities [24]. These small molecules can also exhibit anti-inflammatory effects and protect the host against gut diseases $[25,26]$. Surprisingly, eight core bacteria exhibited potential for SCFA production. Bacteria such as Bacteroides can produce acetate and succinate [27]. Clostridium, Ruminococcus, and Coprococcus are candidate genera associated with butyrate production [10,28]. Streptococcus, which belongs to the order Lactobacillales, can produce acids. Prevotella has an essential role in metabolizing plant cell wall dietary fiber and thus can produce significant amounts of SCFAs [29], consistent with our findings. Prevotella was positively correlated with five core bacteria that all produce SCFAs and are believed to help pigs better adapt to a diet with fiber, which may explain the higher cellulase activity of Hampshire pigs compared with other breeds with a lower abundance of Prevotella. As discussed previously [20], Oscillospira probably produces SCFAs and is negatively associated with inflammatory diseases and body mass index; thus, this genus has been linked to host leanness. The genus Lactobacillus can produce lactic and other acids such as SCFAs and therefore exhibits great importance for host health [2]. However, these bacterial genera require extensive research for both genomic and functional characterization.

\section{CONCLUSION}

Overall, we first compared the fecal microbiota of the four breeds of boar pig by high-throughput sequencing and identified nine core bacteria that may contribute to host digestion and health. Among the nine bacteria, eight genera had the ability to produce SCFAs, and some bacteria can secrete cellulase to aid host fiber digestion. PCoA revealed that Hampshire was separated from the other three breeds, whereas the bacteria of Landrace and Yorkshire were more closely related to each other. Spearman's rank provided valuable information about bacterial competition and cooperation. Several breed-specific bacteria were identified that are likely associated with host genetics and characteristics, but further studies are needed from the physiological perspectives of the host.

\section{CONFLICT OF INTEREST}

We certify that there is no conflict of interest with any financial organization regarding the material discussed in the manuscript.

\section{ACKNOWLEDGMENTS}

The research was supported by the National Natural Science Foundation of China (31402083) and Key project of Natural Science Foundation of Zhejiang Province (LZ15C170001).

\section{REFERENCES}

1.Lu XM, Lu PZ, Zhang H. Bacterial communities in manures of piglets and adult pigs bred with different feeds revealed by $16 \mathrm{~S}$ rDNA 454 
pyrosequencing. Appl Microbiol Biotechnol 2014;98:2657-65.

2. Yang L, Bian G, Su Y, Zhu W. Comparison of faecal microbial community of lantang, bama, erhualian, meishan, xiaomeishan, duroc, landrace, and yorkshire sows. Asian-Australas J Anim Sci 2014;27: 898-906.

3.Kim HB, Isaacson RE. The pig gut microbial diversity: Understanding the pig gut microbial ecology through the next generation high throughput sequencing. Vet Microbiol 2015;177:242-51.

4.Zhang J, Guo Z, Xue Z, et al. A phylo-functional core of gut microbiota in healthy young Chinese cohorts across lifestyles, geography and ethnicities. ISME J 2015;9:1979-90.

5.Bunter K, Bennett C. Genotype comparisons for meat and eating quality traits. Pig Genetics Workshop; 2004.

6.Babol J, Zamaratskaia G, Juneja RK, Lundstrom K. The effect of age on distribution of skatole and indole levels in entire male pigs in four breeds: Yorkshire, Landrace, Hampshire and Duroc. Meat Sci 2004;67:351-8.

7.Pajarillo EA, Chae JP, Balolong MP, et al. Pyrosequencing-based analysis of fecal microbial communities in three purebred pig lines. J Microbiol 2014;52:646-51.

8.Hugerth LW, Wefer HA, Lundin S, et al. DegePrime, a program for degenerate primer design for broad-taxonomic-range PCR in microbial ecology studies. Appl Environ Microbiol 2014;80:5116-23.

9.Schloss PD, Westcott SL, Ryabin T, et al. Introducing mothur: opensource, platform-independent, community-supported software for describing and comparing microbial communities. Appl Environ Microbiol 2009;75:7537-41.

10. Ramayo-Caldas Y, Mach N, Lepage P, et al. Phylogenetic network analysis applied to pig gut microbiota identifies an ecosystem structure linked with growth traits. ISME J 2016;10:2973-7.

11. Lamendella R, Domingo JWS, Ghosh S, Martinson J, Oerther DB. Comparative fecal metagenomics unveils unique functional capacity of the swine gut. BMC Microbiol 2011;11:1-17.

12. Kim HB, Borewicz K, White BA, et al. Longitudinal investigation of the age-related bacterial diversity in the feces of commercial pigs. Vet Microbiol 2011;153:124-33.

13. Miyanaga A, Shimizu K, Noro R, et al. Enterotypes of the human gut microbiome. Nature 2011;473:174-80.

14. Mukherjee PK, Sendid B, Hoarau G, et al. Mycobiota in gastrointestinal diseases. Nat Rev Gastroenterol Hepatol 2015;12:77-87.

15. Hildebrand F, Nguyen TL, Brinkman B, et al. Inflammation-associated enterotypes, host genotype, cage and inter-individual effects drive gut microbiota variation in common laboratory mice. Genome Biol 2013;14:R4.

16. Edward ABP, Jong-Pyo C, Marilen PB, Hyeun BK, Dae-Kyung K. Assessment of fecal bacterial diversity among healthy piglets during the weaning transition. J Gen Appl Microbiol 2014;60:140-6.

17. Pedersen R, Ingerslev HC, Sturek M, et al. Characterisation of gut microbiota in Ossabaw and Gottingen minipigs as models of obesity and metabolic syndrome. PLoS One 2013;8:e56612.

18. Huttenhower C, Gevers D, Knight R, et al. Structure, function and diversity of the healthy human microbiome. Nature 2013;486:207-14.

19. McKnite AM, Perez-Munoz ME, Lu L, et al. Murine gut microbiota is defined by host genetics and modulates variation of metabolic traits. PLoS One 2012;7:e39191.

20. Konikoff T, Gophna U. Oscillospira: a central, enigmatic component of the human gut microbiota. Trends Microbiol 2016;24:523-4.

21. Yang W, Meng F, Peng J, et al. Isolation and identification of a cellulolytic bacterium from the Tibetan pig's intestine and investigation of its cellulase production. Electron J Biotechnol 2014;17:262-7.

22. Kim TH, Kim KS, Choi BH, et al. Genetic structure of pig breeds from Korea and China using microsatellite loci analysis. J Anim Sci 2005;83:2255-63.

23. Scott KP, Duncan SH, Flint HJ. Dietary fibre and the gut microbiota. Anticancer Res 2008;33:201-11.

24. Peng LY, Li ZR, Green RS, Holzman IR, Lin J. Butyrate enhances the intestinal barrier by facilitating tight junction assembly via activation of AMP-activated protein kinase in Caco-2 cell monolayers. J Nutr 2009;139:1619-25.

25. Maslowski KM, Vieira AT, Ng A, et al. Regulation of inflammatory responses by gut microbiota and chemoattractant receptor GPR43. Nature 2009;461:1282-6.

26. Fukuda S, Toh H, Hase K, et al. Bifidobacteria can protect from enteropathogenic infection through production of acetate. Nature 2011;469: 543-7.

27. Shah HN, Collins MD. Proposal to restrict the genus Bacteroides (Castellani and Chalmers) to Bacteroides fragilis and closely related species. Int J Syst Bacteriol 1989;39:85-7.

28. Claesson MJ, Jeffery IB, Conde S, et al. Gut microbiota composition correlates with diet and health in the elderly. Nature 2012;488:178-84.

29. Ellekilde M, Selfjord E, Larsen CS, et al. Transfer of gut microbiota from lean and obese mice to antibiotic-treated mice. Sci Rep 2013;4: 5922. 